\title{
Molecular parity nonconservation in nuclear spin couplings
}

\author{
John W. Blanchard $\odot,{ }^{1, *}$ Jonathan P. King, ${ }^{2,3}$ Tobias F. Sjolander,,${ }^{2,3}$ Mikhail G. Kozlov $\odot,{ }^{4,5}$ and Dmitry Budker $\odot^{1,6,7}$ \\ ${ }^{1}$ Helmholtz-Institut Mainz, GSI Helmholtzzentrum für Schwerionenforschung GmbH, 55128 Mainz, Germany \\ ${ }^{2}$ Department of Chemistry, University of California at Berkeley, Berkeley, California 94720, USA \\ ${ }^{3}$ Materials Science Division, Lawrence Berkeley National Laboratory, Berkeley, California 94720, USA \\ ${ }^{4}$ Petersburg Nuclear Physics Institute of NRC “Kurchatov Institute” Gatchina 188300, Russia \\ ${ }^{5}$ St. Petersburg Electrotechnical University “LETI,” St. Petersburg 197376, Russia \\ ${ }^{6}$ Johannes Gutenberg-Universität Mainz, 55099 Mainz, Germany \\ ${ }^{7}$ Department of Physics, University of California, Berkeley, Berkeley, California 94720-7300, USA
}

(Received 27 February 2020; accepted 29 April 2020; published 1 June 2020)

\begin{abstract}
The weak interaction does not conserve parity, which is apparent in many nuclear and atomic phenomena. However, thus far, parity nonconservation has not been observed in molecules. Here we consider nuclear-spindependent parity-nonconserving contributions to the molecular Hamiltonian. These contributions give rise to a parity-nonconserving indirect nuclear spin-spin coupling which can be distinguished from parity-conserving interactions in molecules of appropriate symmetry, including diatomic molecules. We estimate the magnitude of the coupling, taking into account relativistic corrections. Finally, we propose and simulate an experiment to detect the parity-nonconserving coupling using liquid- or gas-state zero-field nuclear magnetic resonance of electrically oriented molecules and show that ${ }^{1} \mathrm{H}^{19} \mathrm{~F}$ should give signals within the detection limits of current atomic vapor-cell magnetometers.
\end{abstract}

DOI: 10.1103/PhysRevResearch.2.023258

\section{INTRODUCTION}

Parity nonconservation (PNC) in the weak interaction was first theorized [1] in 1956, followed by the first experimental observation in $\beta$ decay of ${ }^{60}$ Co nuclei in 1957 [2]. In the decades since, a variety of parity-nonconserving effects have been observed in atoms $[3,4]$. PNC should also be present in the molecular Hamiltonian $[5,6]$, although its effects have not yet been observed. Molecules afford some interesting possibilities to observe PNC effects, including the proposed detection of energy shifts between enantiomers of chiral molecules [7-17], time-dependent optical activity in chiral molecules [18-20], and Stark interference in linear di- or polyatomic molecules [17,21-23]. Molecules may also provide opportunities to study finer aspects of PNC. In particular, molecules are of interest because of the presence of closely lying levels of opposite parity, which is not a general feature of atoms other than hydrogen [24-26].

Here we propose to observe nuclear-spin-dependent PNC via the indirect nuclear spin-spin coupling in molecules. It has been known for some time that PNC effects should cause frequency shifts between the nuclear magnetic resonance (NMR) spectra of enantiomers of chiral molecules [12,27-36]. Here

\footnotetext{
*blanchard@uni-mainz.de

Published by the American Physical Society under the terms of the Creative Commons Attribution 4.0 International license. Further distribution of this work must maintain attribution to the author(s) and the published article's title, journal citation, and DOI.
}

we consider a different effect which can be observed in a chiral molecules, including diatomic molecules. The indirect nuclear spin-spin coupling ( $J$ coupling) is a commonly measured property in nuclear magnetic resonance spectroscopy [37-39]. The Hamiltonian for two coupled nuclei is bilinear with respect to their spin vector operators $\boldsymbol{I}$ and $\boldsymbol{S}$. The isotropic or scalar $J$ coupling, parametrized by a tensor transforming under rotations as a rank- 0 spherical tensor, is most commonly observed in liquid-state NMR spectroscopy. The corresponding Hamiltonian is

$$
H_{0}=2 \pi J \boldsymbol{I} \cdot \boldsymbol{S} .
$$

In general, the $J$-coupling interaction is characterized by a reducible rank-2 tensor $J_{i k}$ :

$$
H_{J}=2 \pi I_{i} J_{i k} S_{k}
$$

Nonzero-rank contributions to the $J$ coupling can be observed in oriented molecules; for example, a rank-1 coupling can be written as

$$
H_{1}=2 \pi \boldsymbol{J}^{(1)} \cdot \boldsymbol{I} \times \boldsymbol{S},
$$

where $J_{i}^{(1)}=\epsilon_{i j k} J_{j k}$ is a (pseudo)vector when the interaction is P-odd(even), and $\epsilon_{i j k}$ is the Levi-Civita tensor. The effects of $H_{1}$ have not yet been observed, since it averages to zero in isotropically rotating molecules and is suppressed in a magnetic field if spins $I$ and $S$ have different Larmor frequencies. It could, in principle, be observed in solids, but solid-state NMR typically suffers from low resolution. Zero-field nuclear magnetic resonance of electrically oriented molecules can reveal signals from $H_{1}$, as was proposed for absolute determination of molecular chirality [40,41]. Here, 
we propose to apply this technique to observe the interaction $H_{1}$ in a nonchiral molecule, where it can appear due to the contribution of the nuclear-spin-dependent parity-nonconserving weak interaction [27].

For oriented molecules, the residual component of $\boldsymbol{J}^{(1)}$ must be parallel to the orientation axis. A paritynonconserving $\boldsymbol{J}^{(1)}$ with nonzero projection along the molecular electric dipole can be found in molecules belonging to several symmetry point groups (see Supplemental Material [42]), including diatomics $\left(C_{\infty v}\right)$. Of course, diatomics are nonchiral and, therefore, there can be no observable parityconserving contribution to $\boldsymbol{J}^{(1)}$.

There is a similarity between the effect considered here and the hyperfine correction to the nuclear-spin-independent PNC interaction, which mimics the nuclear-spin-dependent PNC interaction $[43,44]$. There both interactions are centered on the same nucleus. Since these interactions diverge at the origin, one ends up with a highly singular effective operator, which is difficult to calculate accurately. Here we take the hyperfine interaction and nuclear-spin-dependent PNC interaction on different nuclei. This gives us the PNC spin-spin coupling, which is described by a less singular effective operator that may be easier to calculate (see below).

\section{THEORY}

We now estimate the magnitude of $\boldsymbol{J}_{\mathrm{PNC}}^{(1)}$ in the diatomics ${ }^{205} \mathrm{Tl}{ }^{19} \mathrm{~F}$ and ${ }^{1} \mathrm{H}^{19} \mathrm{~F}$. $\boldsymbol{J}_{\mathrm{PNC}}^{(1)}$ arises from the nuclear-spindependent parity-odd weak interaction. For a first-order approximation, we include only terms that are linear in the nuclear spin operators $\boldsymbol{I}_{K}$ (we use the notation $\boldsymbol{I}_{K}$ for a generic nuclear spin operator but write $\boldsymbol{I}$ and $\boldsymbol{S}$ when explicitly considering a two-spin Hamiltonian for calculation of observables in an NMR experiment). We also neglect terms that contain electron-spin operators since, to a first-order approximation, they do not contribute to PNC in the diamagnetic molecules under consideration [26].

In the nonrelativistic approximation the Hamiltonian for the $\boldsymbol{J}^{(1)}$ parity-nonconserving interaction has the form (using atomic units $\left.\hbar=m_{e}=|e|=1\right)$

$$
H_{\mathrm{PNC}}=\frac{G \alpha}{2 \sqrt{2}} \sum_{i, K} g_{K} \boldsymbol{I}_{K}\left[\boldsymbol{p}_{i}, \delta\left(\boldsymbol{r}_{i}-\boldsymbol{R}_{K}\right)\right]_{+},
$$

where $G \approx 2.22 \times 10^{-14}$ is the Fermi constant, $\alpha \approx 1 / 137$ is the fine-structure constant, $\boldsymbol{p}_{i}=-i \nabla_{i}$ and $\boldsymbol{r}_{i}$ are the momentum and coordinate of the $i$ th electron, and $\boldsymbol{R}_{K}$ is the coordinate of the nucleus $K$. The dimensionless coupling constant $g_{K}$ is of order unity. Two main contributions to this coupling come from the nuclear anapole moment [45] and the electroweak electron vector and nucleon axial-vector interaction [26]. For the heavy nuclei the anapole contribution usually dominates [21,46,47].

To account for a magnetic field, we substitute the canonical momentum [48]

$$
\boldsymbol{p}_{i} \rightarrow \boldsymbol{\pi}_{i}=\boldsymbol{p}_{i}+\alpha \boldsymbol{A},
$$

where $\boldsymbol{A}$ is the vector potential. In the case of spin-spin coupling, the magnetic field is produced by the magnetic moment of nucleus $L, \boldsymbol{\mu}_{L}=\gamma_{L} \boldsymbol{I}_{L}$, where $\gamma_{L}$ is the gyromagnetic ratio. We can then take

$$
\boldsymbol{A}_{L}=\gamma_{L} \frac{\boldsymbol{I}_{L} \times\left(\boldsymbol{r}-\boldsymbol{R}_{L}\right)}{\left|\boldsymbol{r}-\boldsymbol{R}_{L}\right|^{3}} .
$$

Substituting Eqs. (6) and (5) into Eq. (4), we obtain

$$
\begin{aligned}
H_{\mathrm{PNC}}= & \frac{G \alpha}{2 \sqrt{2}} \sum_{i, K} g_{K} \boldsymbol{I}_{K}\left[\boldsymbol{p}_{i}, \delta\left(\boldsymbol{r}_{i}-\boldsymbol{R}_{K}\right)\right]_{+} \\
& +\frac{G \alpha^{2}}{\sqrt{2}} \sum_{i, K, L} g_{K} \gamma_{L} \frac{\boldsymbol{I}_{K} \cdot\left[\boldsymbol{I}_{L} \times\left(\boldsymbol{R}_{K}-\boldsymbol{R}_{L}\right)\right]}{\left|\boldsymbol{R}_{K}-\boldsymbol{R}_{L}\right|^{3}} \delta\left(\boldsymbol{r}_{i}-\boldsymbol{R}_{K}\right) .
\end{aligned}
$$

The second term here is bilinear in nuclear spin operators and contributes to the spin-spin coupling [27]. By comparison to Eq. (3) we obtain $\boldsymbol{J}_{\mathrm{PNC}}^{(1)}$ in vector form:

$$
\boldsymbol{J}_{\mathrm{PNC}}^{(1)}=\frac{G \alpha^{2}}{2 \pi \sqrt{2}}\left(\gamma_{S} g_{I}\left\langle\Psi_{e}\left|\sum_{i} \delta\left(\boldsymbol{r}_{i}-\boldsymbol{R}_{I}\right)\right| \Psi_{e}\right\rangle-g_{S} \gamma_{I}\left\langle\Psi_{e}\left|\sum_{i} \delta\left(\boldsymbol{r}_{i}-\boldsymbol{R}_{S}\right)\right| \Psi_{e}\right\rangle\right) \frac{\boldsymbol{R}_{I}-\boldsymbol{R}_{S}}{\left|\boldsymbol{R}_{I}-\boldsymbol{R}_{S}\right|^{3}} .
$$

The electronic matrix elements in (8) correspond to the total electronic densities on the respective nuclei:

$$
\left\langle\Psi_{e}\left|\sum_{i} \delta\left(\boldsymbol{r}_{i}-\boldsymbol{R}_{K}\right)\right| \Psi_{e}\right\rangle=\rho_{e}\left(\boldsymbol{R}_{K}\right) .
$$

Substituting into (8) we obtain

$$
\boldsymbol{J}_{\mathrm{PNC}}^{(1)}=\frac{G \alpha^{2}}{2 \pi \sqrt{2}}\left(\gamma_{S} g_{I} \rho_{e}\left(\boldsymbol{R}_{I}\right)-g_{S} \gamma_{I} \rho_{e}\left(\boldsymbol{R}_{S}\right)\right) \frac{\boldsymbol{R}_{I, S}}{\left|\boldsymbol{R}_{I, S}\right|^{3}},
$$

where $\boldsymbol{R}_{I, S}=\boldsymbol{R}_{I}-\boldsymbol{R}_{S}$. The dominant contribution to the density (9) comes from the two $K$-shell electrons, whose wave functions are hydrogenic [49], $\psi_{1 s} \approx 2 \sqrt{Z^{3} / 4 \pi} e^{-Z r}$; therefore

$$
\rho_{e}\left(\boldsymbol{R}_{K}\right) \approx \frac{2}{\pi} Z_{K}^{3} .
$$

The contribution of the $2 s$ shell is approximately eight times smaller and can be neglected in the estimates (for hydrogen, the density at the origin scales as $1 / n^{3}$ ). Putting (11) in (10) we arrive at

$$
\boldsymbol{J}_{\mathrm{PNC}}^{(1)}=\frac{G \alpha^{2}}{\pi^{2} \sqrt{2}}\left(\gamma_{S} g_{I} Z_{I}^{3}-g_{S} \gamma_{I} Z_{S}^{3}\right) \frac{\boldsymbol{R}_{I, S}}{\left|\boldsymbol{R}_{I, S}\right|^{3}} .
$$

Typical internuclear distances $R_{I, S}$ are comparable to the bond length and are about 3-4 Bohr radii. Given the $Z^{3}$ scaling, the term in parentheses in Eq. (10), including $Z$ from the heaviest atom, will dominate. Assuming $R_{I, S} \approx 4$, the 
magnitude of $\boldsymbol{J}_{\mathrm{PNC}}^{(1)}$ is estimated:

$$
J_{\mathrm{PNC}}^{(1)} \sim \frac{G \alpha^{2}}{16 \pi^{2} \sqrt{2}} \gamma_{S} g_{I} Z_{I}^{3},
$$

where $Z_{I}$ is the charge of the heaviest nucleus in the molecule.

For heavier atoms, relativistic effects become important. The relativistic enhancement factor is (Supplemental Material) $[26,50]$

$$
F_{\mathrm{rel}}=\frac{2(1+\gamma)\left(2 Z R_{\mathrm{nuc}}\right)^{2 \gamma-2}}{\Gamma^{2}(2 \gamma+1)}, \gamma=\sqrt{1-(\alpha Z)^{2}},
$$

where $R_{\text {nuc }}$ is the nuclear radius in atomic units and $\Gamma$ refers to a gamma function. Equation (12) then becomes

$$
\boldsymbol{J}_{\mathrm{PNC}}^{(1)}=\frac{G \alpha^{2}}{\pi^{2} \sqrt{2}}\left(\gamma_{S} g_{I} Z_{I}^{3} F_{\mathrm{rel}, I}-g_{S} \gamma_{I} Z_{S}^{3} F_{\mathrm{rel}, S}\right) \frac{\boldsymbol{R}_{I, S}}{\left|\boldsymbol{R}_{I, S}\right|^{3}} .
$$

\section{A. Estimates}

Let us estimate the magnitude $J_{\mathrm{PNC}}^{(1)}$ for the molecules ${ }^{205} \mathrm{Tl}{ }^{19} \mathrm{~F}$ and ${ }^{1} \mathrm{H}^{19} \mathrm{~F}$. TIF is a popular candidate for molecular tests of fundamental symmetries because the heavy ${ }^{205} \mathrm{Tl}$ atom $(Z=81)$ is expected to give rise to strong $\mathrm{P}$ - and T-violating effects [4,51-54]. From Eq. (15), neglecting contribution of the fluorine and using internuclear distance $R=3.93$ a.u. and relativistic factor $F_{\text {rel,Tl }}=7.6$, we obtain

$$
J_{\mathrm{PNC}, \mathrm{TIF}}^{(1)} \approx 9 \times 10^{-3} g_{\mathrm{Tl}} \mathrm{Hz} .
$$

According to Flambaum et al. [46], the coupling constant for $\mathrm{Tl}$ is $g_{\mathrm{Tl}} \approx 0.5$. While this value is promising given the resolution of zero-field NMR, diatomic TIF does not exist in the liquid phase and zero-field NMR would be a significant experimental challenge. A molecular-beam experiment featuring electric-field orientation could be a good option for TIF [55]. Indeed, hyperfine measurements with molecular beams have been used to determine the scalar and symmetric rank-2 components of the TIF $J$ coupling [56,57].

Let us estimate the PNC coupling for ${ }^{1} \mathrm{H}^{19} \mathrm{~F}$. The relativistic factor (14) for fluorine is very close to unity, so we can use the nonrelativistic expression (12), which gives

$$
J_{\mathrm{PNC}, \mathrm{HF}}^{(1)} \approx 9 \times 10^{-6} g_{\mathrm{F}} \mathrm{Hz} .
$$

Liquid $\mathrm{HF}$ would be an option; as the neat liquid has a concentration of $\sim 50 \mathrm{M}$ at its boiling point $\left(19.5^{\circ} \mathrm{C}\right)$, both ${ }^{1} \mathrm{H}$ and ${ }^{19} \mathrm{~F}$ are naturally abundant spin- $1 / 2$ isotopes, and the molecular dipole moment of $1.86 \mathrm{D}$ is reasonably large (a large dipole moment is needed to achieve a high degree of molecular orientation). Unfortunately, due to hydrogenbonding and autoionization effects, $J_{\mathrm{HF}}$ can only be observed when $\mathrm{HF}$ is diluted in aprotic solvents or when the neat liquid is cooled to very low temperatures [58-60].

A possible alternative could be the endofullerene complex HF@ $\mathrm{C}_{60}$ [61], though the molecular dipole moment is decreased to $0.45 \mathrm{D}$, and in order to avoid broadening due to intermolecular dipole-dipole couplings, it would likely be necessary to dissolve the HF@ $\mathrm{C}_{60}$ in a solvent such as 1chloronaphthalene-this would result in an overall decrease in concentration by some three orders of magnitude. More suitable from an experimental standpoint may be liquid or gaseous fluoromethane $\left(\mathrm{CH}_{3} \mathrm{~F}\right)$, which has a molecular dipole moment of $1.81 \mathrm{D}$ and a concentration of $\sim 16 \mathrm{M}$ at its saturation pressure of $33 \mathrm{bar}$ at $25^{\circ} \mathrm{C}$. The magnitude of the PNC $J$ couplings for this molecule are estimated in the microhertz range. ${ }^{1}$

$\boldsymbol{J}_{\mathrm{PNC}}^{(1)}$ is fixed with respect to the molecular orientation, but small molecules in the liquid or gas state undergo fast molecular rotation. In NMR, we measure the averaged $\overline{\boldsymbol{J}_{\mathrm{PNC}}^{(1)}}$, which is nonzero only when the molecules are oriented. The residual rank-1 coupling for a molecule oriented in an electric field is [62]

$$
\overline{J^{(1)}}=\frac{d E}{3 k T} J^{(1)},
$$

where $d$ is the electric dipole moment, $E$ is the electric field experienced by the molecule, $k$ is the Boltzmann constant, and $T$ the temperature. The scaling factor is thus given by $d E / 3 k T$. In polar liquids $E$ is related to the macroscopic electric field as $E \approx\left[\left(\epsilon_{r}+2\right) / 3\right] E_{\mathrm{mac}}$, where $\epsilon_{r}$ is the static relative permittivity of the medium. For HF, $d=1.86 \mathrm{D}$, and $\epsilon_{r}=84$. Assuming a temperature of $300 \mathrm{~K}$ and $E_{\mathrm{mac}} \approx$ $70 \mathrm{kV} / \mathrm{cm}$ [63], the orientation scaling factor is 0.1 . This gives $\overline{J_{\mathrm{PNC}, \mathrm{HF}}^{(1)}} \approx 1 \times 10^{-6} \mathrm{~Hz}$ for the estimated projection of $\overline{J_{\mathrm{PNC}, \mathrm{HF}}^{(1)}}$ along the $z$ axis.

\section{B. Spin dynamics}

Zero-field NMR involves measuring the evolution of nuclear magnetism of coupled spins in the absence of external magnetic fields [64] [Fig. 1(a)]. Signals are typically detected with an atomic vapor-cell magnetometer. When a diatomic molecule is oriented along the $z$ axis, the nuclear spin Hamiltonian is

$$
\frac{H}{2 \pi}=J \boldsymbol{I} \cdot \boldsymbol{S}+\frac{\overline{i J_{z}^{(1)}}}{2}\left(I^{+} S^{-}-I^{-} S^{+}\right)-\bar{D}\left(3 I_{z} S_{z}-\boldsymbol{I} \cdot \boldsymbol{S}\right),
$$

where the residual dipolar coupling [62] is

$$
\bar{D}=\frac{\gamma_{I} \gamma_{S}}{r^{3}} \frac{1}{30}\left(\frac{d E}{k T}\right)^{2} .
$$

\footnotetext{
${ }^{1}$ The $\mathrm{CH}_{3} \mathrm{~F}$ molecule can have three different couplings- $\mathrm{HC}$, HF, and CF. For the HF coupling we can use the same estimate as for the HF molecule and scale it by the square of the distance $R_{H F}$, which is here 3.8 a.u. instead of 1.7 a.u. This gives us $9 \mu \mathrm{Hz} \times(1.7 / 3.8)^{2} \approx$ $2 \mu \mathrm{Hz}$. For the $\mathrm{HC}$ coupling in addition to the distance $(2.05)$ we need to change the charge, so we get $9 \mu \mathrm{Hz} \times(1.7 / 2.1)^{2} \times(6 / 9)^{3} \approx$ $2 \mu \mathrm{Hz}$. Thus, both couplings are of the same size and about five times smaller than in HF. The coupling of $\mathrm{C}$ and $\mathrm{F}$ is different. Here we have both terms in Eq. (15) and they almost cancel each other, so the result is much smaller and unreliable. If we polarize the molecule in an electric field, we need to project the vector $\boldsymbol{J}^{(1)}$ on the molecular axis. This will give us additional suppression by $\cos \phi$, where $\phi$ is the angle between the molecular axis and $\boldsymbol{J}^{(1)}$. For $\mathrm{HC} \cos \phi \approx 0.3$, and for $\mathrm{HF} \cos \phi \approx 0.8$. All in all, we get close to $1 \mu \mathrm{Hz}$ and $2 \mu \mathrm{Hz}$ for HC and HF PNC couplings, respectively.
} 


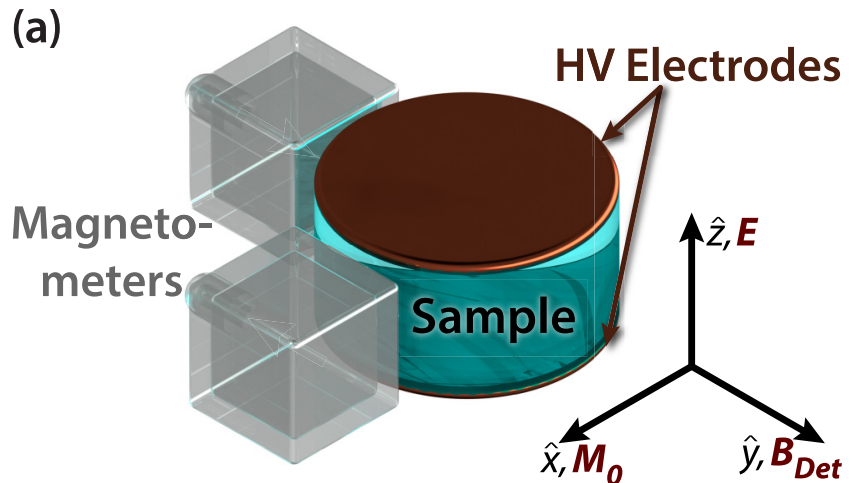

(b)

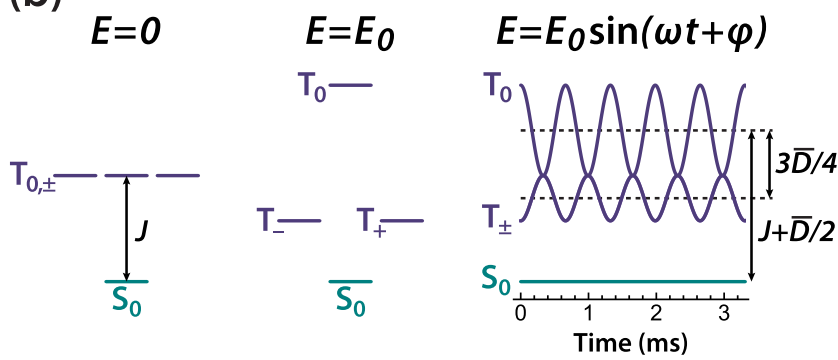

FIG. 1. Experimental arrangement. (a) The apparatus includes a sample made of polar achiral molecules, each of which is a two-spin heteronuclear spin system oriented by an electric field $\boldsymbol{E}$ produced by high-voltage (HV) electrodes. The nuclear spin state is prepared such that the initial magnetization is oriented along $\boldsymbol{M}_{0}$, and magnetometers (depicted here as glass vapor cells) are used to measure the nuclear spin magnetization projection along $\boldsymbol{B}_{\text {Det }}$. (b) Nuclear spin eigenstates for various electric-field conditions. In the absence of any applied fields are three degenerate triplet states and a singlet state, separated by the $J$ coupling. Application of a dc electric field introduces a residual dipole-dipole coupling of strength $\bar{D}$ that shifts the $\left|T_{0}\right\rangle$ state by $\bar{D}$ and the $\left|T_{ \pm}\right\rangle$states by $-\bar{D} / 2$. An ac electric field modulates the triplet states such that the average $\left|S_{0}\right\rangle \leftrightarrow$ $\left|T_{0}\right\rangle$ separation is $J+\bar{D} / 2$ and the average $\left|T_{0}\right\rangle \leftrightarrow\left|T_{ \pm}\right\rangle$separation is $3 \bar{D} / 4$.

Rapid motion around the $z$ axis averages components of $\overline{J^{(1)}}$ and $\bar{D}$ orthogonal to the $z$ axis to zero. $J$ is the scalar component of the $J$ coupling, which is not affected by molecular rotation. Even though $\bar{D}$ scales quadratically with the electric field, it cannot be neglected, as the dipolar coupling for a HF molecule fully aligned along $z$ is approximately $150 \mathrm{kHz}$. For the conditions described above, the residual coupling $\bar{D}=452 \mathrm{~Hz}$, which is comparable to the isotropic $J$ coupling, $\sim 530 \mathrm{~Hz}$ [65]. The $z$ component of the symmetric anisotropic $J$ coupling has a form identical to that of the dipolar coupling and effectively adds to $\bar{D}$, but it is a small effect that can be neglected for the present analysis.

In the absence of electric fields, the nuclear spin eigenstates are the singlet $(|S\rangle)$ and three degenerate triplet states $\left(\left|T_{0, \pm 1}\right\rangle\right)$, with an energy separation equal to $J$. The dipolar coupling shifts the $\left|T_{0}\right\rangle$ state by $\bar{D}$ and the $\left|T_{ \pm 1}\right\rangle$ states by $-\bar{D} / 2$ [66].

If an oscillating electric field at a frequency $\omega$ is applied, the term proportional to $J_{\mathrm{PNC}}$ is modulated at this frequency, and the term proportional to $\bar{D}$ is modulated at twice this frequency:

$$
\begin{aligned}
\frac{H}{2 \pi}= & J \boldsymbol{I} \cdot \boldsymbol{S}+\frac{i \sin (\omega t+\phi) \overline{J_{\mathrm{PNC}}}}{2}\left(I^{+} S^{-}-I^{-} S^{+}\right) \\
& -\frac{1-\cos (2 \omega t+2 \phi)}{2} \bar{D}\left(3 I_{z} S_{z}-\boldsymbol{I} \cdot \boldsymbol{S}\right),
\end{aligned}
$$

where $\phi$ is the phase of the ac electric field, and $\overline{J_{\mathrm{PNC}}}$ and $\bar{D}$ now refer to the peak values. Gathering terms based on time dependence, we find

$$
\begin{aligned}
\frac{H}{2 \pi}= & \left(J+\frac{\bar{D}}{2}\right) \boldsymbol{I} \cdot \boldsymbol{S}-\frac{3 \bar{D}}{2} I_{z} S_{z} \\
& +\frac{i \sin (\omega t+\phi) \overline{J_{\mathrm{PNC}}}}{2}\left(I^{+} S^{-}-I^{-} S^{+}\right) \\
& -\frac{\cos (2 \omega t+2 \phi)}{2} \bar{D}\left(3 I_{z} S_{z}-\boldsymbol{I} \cdot \boldsymbol{S}\right) .
\end{aligned}
$$

To gain a physical intuition regarding this system, it is helpful to first consider the case of no interaction other than the scalar $J$ coupling. Suppose we begin by preparing an initial state in which the spins $I$ and $S$ have opposite average projection onto some direction $(\hat{x})$ corresponding to magnetization $\boldsymbol{M}_{0}$ along this direction (the sample is at zero external magnetic field), see Fig. 2(a).

The effect of the $J$ coupling (i.e., the $\boldsymbol{I} \cdot \boldsymbol{S}$ interaction) is to produce beats of the spins so that the average values of $\boldsymbol{I}$ and $\boldsymbol{S}$ oscillate between positive and negative values and the magnetization $\boldsymbol{M}$ correspondingly oscillates along $\hat{x}$. At the times when the average values of $\boldsymbol{I}, \boldsymbol{S}$, and $\boldsymbol{M}$ go through zero, the polarization of the spin system has higher order and the evolution is akin to the process of alignment-to-orientation conversion known in atomic and molecular physics [67]. Interpreting the evolution in the product-operator formalism [68], the isotropic $J$ coupling converts $I_{x}-S_{x}$ into $I_{y} S_{z}-I_{z} S_{y}$, then into $S_{x}-I_{x}$, etc., as depicted in Fig. 2(a).

Now let us add an electric field $\vec{E}$ applied along $\hat{z}$ perpendicular to $\boldsymbol{M}_{0}$, which orients the molecules, giving rise to nonzero $\overline{\boldsymbol{J}^{(1)}}$ [see Fig. 1(a)]. In the absence of other interactions, the effect of $\overline{J^{(1)}}$ would be to convert $I_{x}-S_{x}$ into $I_{y} S_{z}+$ $I_{z} S_{y}$, then into $S_{x}-I_{x}$, etc., which would not produce any magnetization effects distinguishable from those of isotropic $J$.

An "interesting" effect of $\overline{\boldsymbol{J}^{(1)}}$ appears when this coupling is combined with the scalar part to convert $I_{y} S_{z}-I_{z} S_{y}$ generated by the latter into $I_{y}+S_{y}$ associated with a detectable magnetization along $\hat{y}$ [see Fig. 2(b) and Appendix for details].

The essential observable phenomenon revealing the rank-1 $J$ coupling in the system is the component of the magnetization perpendicular to both $\overline{\boldsymbol{J}^{(1)}}$ and the original direction of the magnetization. In fundamental physics experiments, it is customary to consider a rotational invariant, a (pseudo)scalar built out of experimental parameters that reveals the symmetry of the effect and the behavior of the system under the reversals of the parameters. In this case, we can write such an invariant as

$$
\boldsymbol{B}_{\text {Det }} \cdot \boldsymbol{M}_{0} \times \boldsymbol{E},
$$


(a)

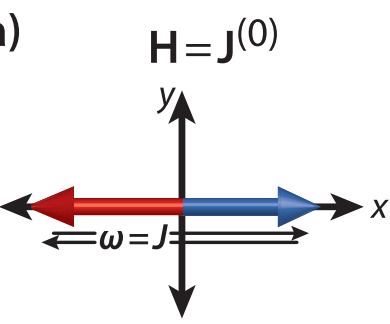

(b)

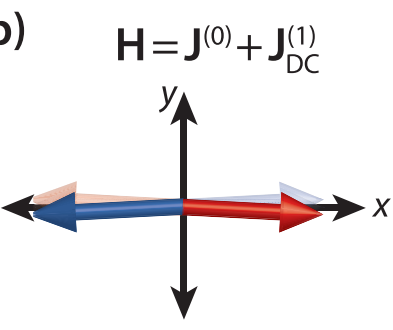

(c)

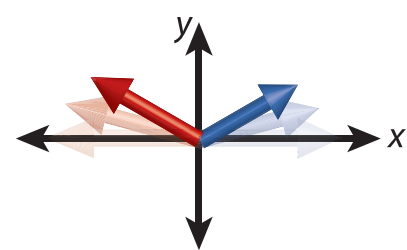

(d)

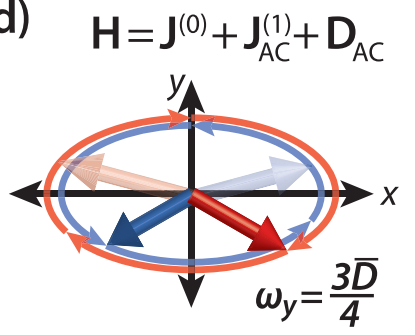

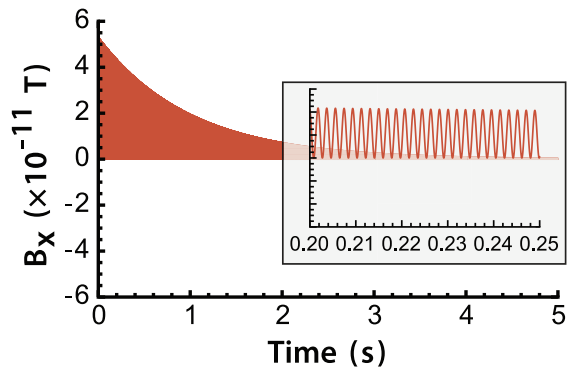
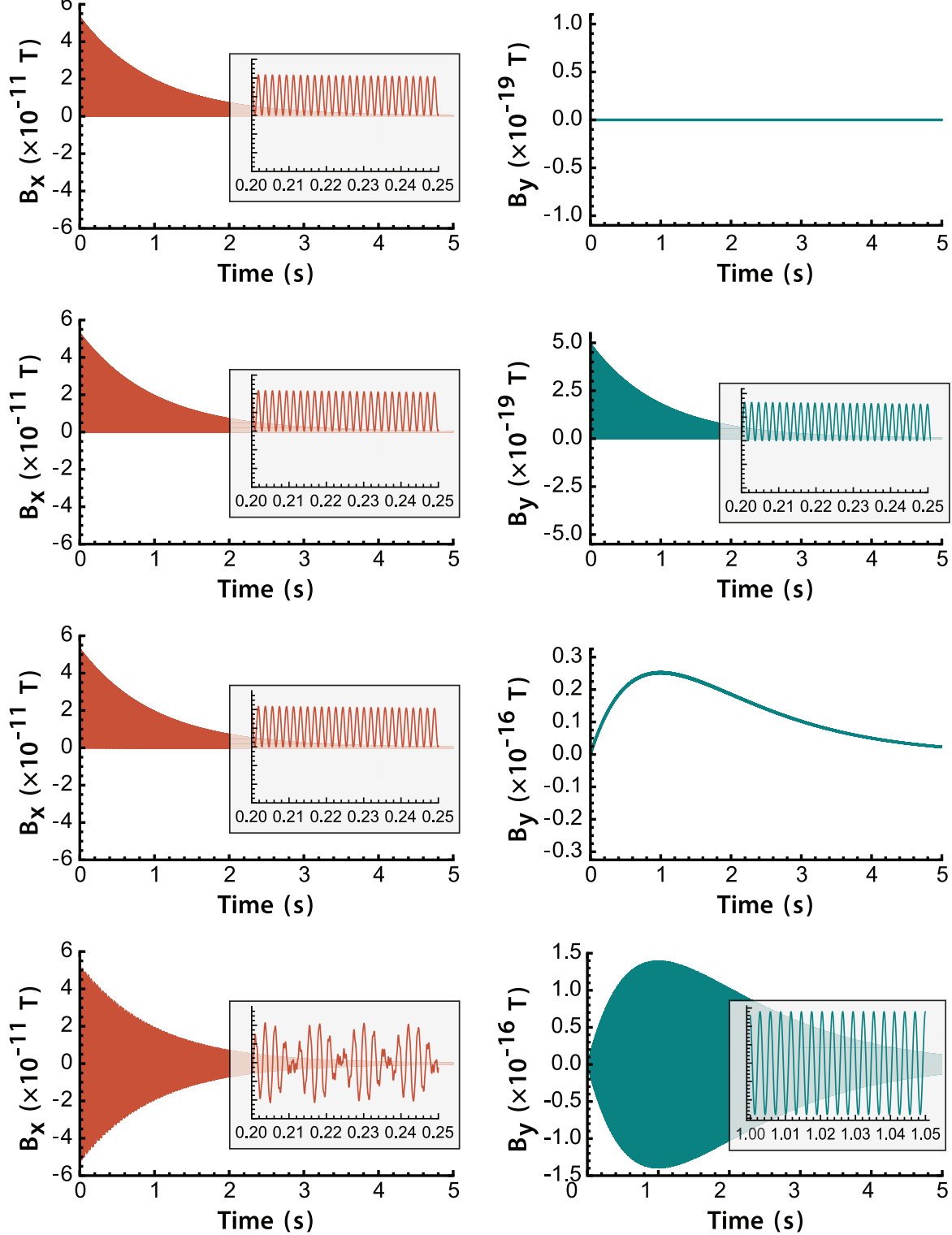

FIG. 2. Evolution of nuclear magnetization at zero magnetic field. Vector diagrams show the evolution of $\boldsymbol{I}$ and $\boldsymbol{S}$ magnetization, starting from $I_{x}-S_{x}$. Simulated signals along $\hat{x}$ and $\hat{y}$, assuming an initial state proportional to $\gamma_{I} I_{x}-\gamma_{S} S_{x}$. (a) Evolution under the isotropic $J$ coupling. (b) Including a static $\overline{J_{z}^{(1)}}$. (c) With a resonantly modulated $\overline{J_{z}^{(1)}}$. (d) Including the residual dipole-dipole coupling due to alignment by the electric field. In all cases, the spins evolve only in the $\hat{x} \hat{y}$ plane.

which is P-odd and T-even (i.e., a pseudoscalar invariant with respect to time reversal), revealing that the relevant signal should reverse sign under the reversals of each of the quantities $\boldsymbol{B}_{\text {Det }}, \boldsymbol{M}_{0}$, and $\boldsymbol{E}$.

We now discuss the time dependence of the signal that can be evaluated based on Eq. (22) and the initial conditions. Let us first consider just the scalar $J$ coupling and neglect the other coupling. As illustrated in Fig. 2(a), there is magnetization only along the initial direction, which undergoes oscillations at frequency $J$. Adding the antisymmetric $J$ coupling, we see that there appears a transverse component of magnetization along the detection direction oscillating at the same frequency Fig. 2(b). The maximum transverse magnetization can be roughly estimated as $M_{0} \overline{J^{(1)}} / J$.
The effect may be considerably enhanced by a factor on the order of $J T_{2}$ if we reverse the direction of the electric field synchronously with the reversals of the main component of the magnetization, Fig. 2(c).

Finally, we reintroduce the dipole-dipole coupling. The $\boldsymbol{I} \cdot \boldsymbol{S}$ part of the interaction [see Eq. (22)] adds to the scalar $J$ coupling and does not bring any qualitative new features, except for changing the frequency of the oscillation of the main component of the magnetization to

$$
\omega / 2 \pi=J+\bar{D} / 2 \text {. }
$$

On the other hand, interestingly, the $I_{z} S_{z}$ term results in an enhancement of the signal associated with antisymmetric $J$ coupling, as illustrated in Fig. 2(d). Moreover, the PNC-induced $y$ magnetization is no longer static. Because the singlet spin 
state commutes with $I_{z} S_{z}$, the induced evolution must involve only the triplet states, so it oscillates at frequency $3 \bar{D} / 4$, corresponding to the average energy difference between the $\left|T_{0}\right\rangle$ and $\left|T_{ \pm 1}\right\rangle$ states. Importantly, this frequency is distinct from the frequency with which the electric field is modulated $(J+\bar{D} / 2)$, provided that $J \neq \bar{D} / 4$.

\section{THE PROPOSED EXPERIMENT}

Our proposed experiment involves excitation of magnetization along the $y$ axis. An experimentally realizable initial condition is

$$
\rho(0)=\frac{1}{4}+\frac{B_{\mathrm{p}}}{4 k T}\left[\frac{\gamma_{I}+\gamma_{S}}{2}\left(I_{x}+S_{x}\right)+\frac{\gamma_{I}-\gamma_{S}}{2}\left(I_{x}-S_{x}\right)\right],
$$

which corresponds to magnetizing the spins along the $x$ axis in a field of strength $B_{\mathrm{p}}$ (where $\left|\gamma B_{\mathrm{p}}\right| \gg|2 \pi J|$ ) at a temperature $T ; k$ is Boltzmann's constant. Following prepolarization, the sample is transported to the detection region in the presence of a guiding magnetic field, $S$ is inverted, and then the guiding field is suddenly turned off. The inversion of $S$ can be done either in high field [69] or at zero field [70]; the purpose of the inversion is to maximize $I_{x}-S_{x}$, and, correspondingly, the useful signal.

When $J_{\mathrm{PNC}} \neq 0$, there is nonzero magnetization along $\hat{y}$ that oscillates at frequency $3 \bar{D} / 4$ [see Fig. 2(d)]. Figure 2 shows a simulation of the PNC-dependent signal for ${ }^{1} \mathrm{H}^{19} \mathrm{~F}$. We assume the spins are prepolarized in a field $B_{p}=20 \mathrm{~T}$ at $300 \mathrm{~K}$ and that the spin coherence time is $1 \mathrm{~s}$. The simulated sample is $10^{21}$ molecules $(50 \mu \mathrm{L})$ at a distance of $7 \mathrm{~mm}$ from the magnetometer cell, which is typical of zero-field NMR detection. Figure 2(d) shows the time evolution of the magnetic field at the magnetometer cell, which has an amplitude of $\approx 1.5 \times 10^{-16} \mathrm{~T}$. Given a magnetometer sensitivity of $10^{-14} \mathrm{~T} / \sqrt{\mathrm{Hz}}$ and a realistic duty cycle of $10 \%$, this would require on the order of hours to achieve a signal-tonoise ratio greater than unity. We emphasize that this level of signal corresponds to readily accessible laboratory conditions similar to those in zero-field spectrometers currently in use $[64,71,72]$.

Note the beating in the $\hat{x}$ signal [Fig. 2(d)] that does not appear in the parity-violation-related $\hat{y}$ signal. The origin of the beating is that the $\hat{x}$ signal has a component at a second frequency corresponding to the $\left|S_{0}\right\rangle \leftrightarrow\left|T_{ \pm 1}\right\rangle$ energy interval, $J-\bar{D} / 4$. This component arises from the $I_{x}-S_{x}$ component of the initial state. Because the $\hat{x}$ component is several orders of magnitude larger than the $\hat{y}$ component, in a realistic experiment there will be inevitable "leakage" of the $\hat{x}$ signal into the $\hat{y}$ channel. However, the distinct time dependence of the former will allow one to subtract (i.e., "fit out") the accurately measured $\hat{x}$ signal from the $\hat{y}$ channel, facilitating the discrimination of the sought-after signal.

\section{Systematic effects}

Systematic errors can be addressed by considering how they are affected by reversals of the electric field $\boldsymbol{E}$, the initial nuclear magnetization $\boldsymbol{M}_{0}$, and the sensitive axis of the detector, $\boldsymbol{B}_{\text {Det }}$, considering that the PNC signal transforms as the rotational invariant of Eq. (23).
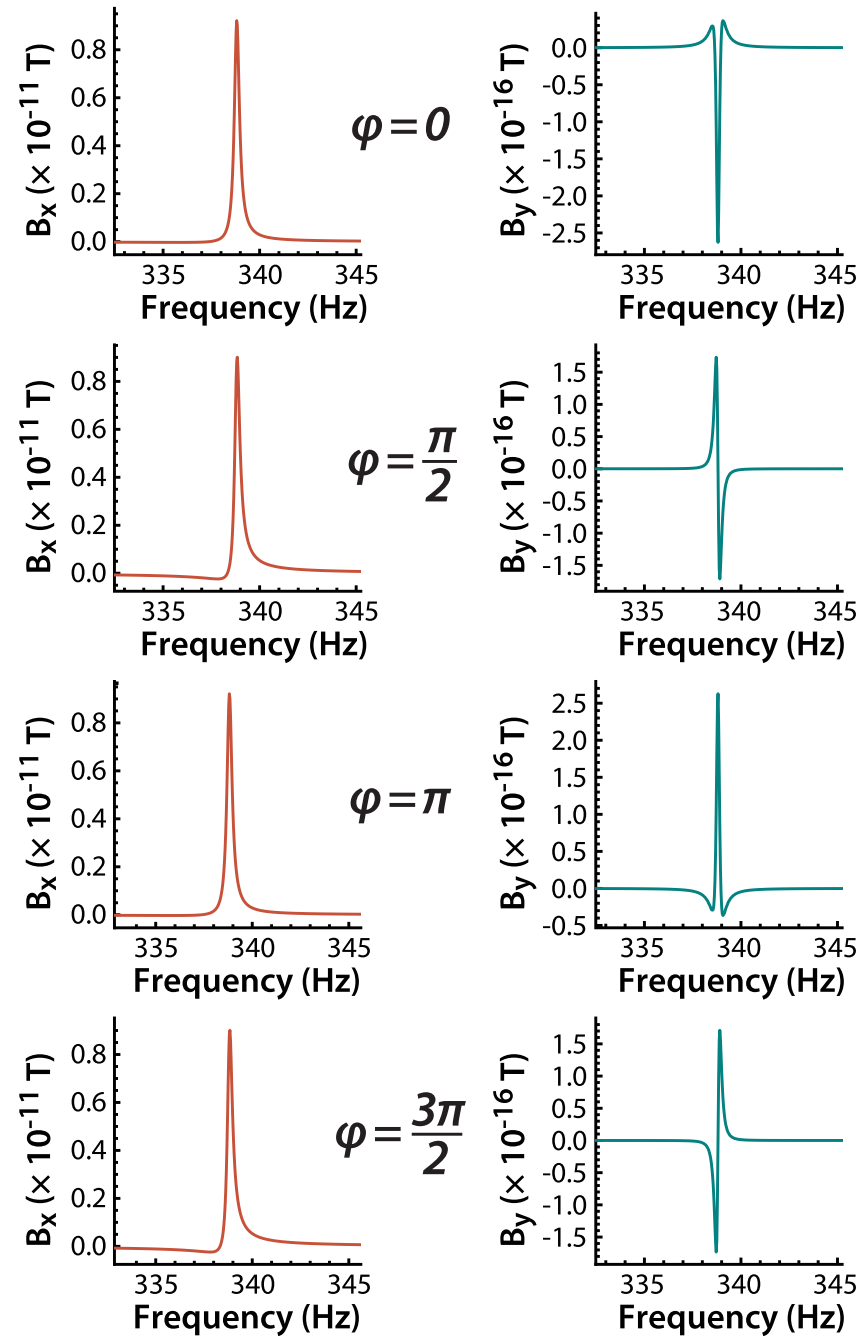

FIG. 3. Electric-field phase cycling. Dependence of $B_{x}$ (parityconserving) and $B_{y}$ (PNC) signals on the phase of the electric-field modulation.

Our method has the advantage that the PNC signal will emerge at the frequency $3 \bar{D} / 4$, which is different from that of the driving electric field $(J+\bar{D} / 2)$. However, a component of the parity-conserving NMR signal also evolves at this frequency, with amplitude orders of magnitude larger than that of the PNC signal, and it is likely impossible to avoid slight misalignments that would result in the magnetometer signal having at least some component of the parity-conserving signal added to the PNC signal. Fortunately, the parity-conserving and nonconserving signals depend differently on the phase of the oscillating electric field. Indeed, the phase of the PNC signal depends linearly on the phase of the electric field, while the parity-conserving signal is invariant under reversal of the phase (see Fig. 3). The PNC signal can thus be isolated by performing subsequent experiments with the electric-field orientation reversed (a $180^{\circ}$ phase shift) and subtracting the signals.

In the design of the experiments, in addition to misalignment, one needs to consider also the effect of the ac magnetic field $\boldsymbol{B}_{a c}$ that would inevitably accompany the oscillating electric field. One example of a potentially dangerous systematic 
error corresponds to the rotational invariant $\boldsymbol{B}_{\text {Det }} \cdot \boldsymbol{B}_{a c}$. Indeed, this signal reverses with the reversal of the applied electric field (which is the origin of $\boldsymbol{B}_{a c}$ ) and the detection direction. However, this particular effect can be discriminated based on the fact that it does not reverse with the initial magnetization of the sample.

Other potential systematic effects may arise due to the presence of stray magnetic fields. Perhaps the most concerning issue is that an ac magnetic field at the frequency of the electric-field modulation and directed along the $\hat{z}$ axis has a very similar effect to that of $\overline{J_{\mathrm{PNC}}}$, generating $I_{y}+S_{y}$ spin order, and thus an oscillating magnetization along $\hat{y}$ at the frequency $3 \bar{D} / 4$. For a ${ }^{1} \mathrm{H}-{ }^{19} \mathrm{~F}$ spin system, $B_{z}(t) \approx 100 \mathrm{fT}$ yields an effect of the same magnitude as $\overline{\boldsymbol{J}^{(1)}}$ under the conditions discussed above. This stray-field-induced systematic signal transforms as the rotational invariant

$$
\boldsymbol{B}_{\text {Det }} \cdot \boldsymbol{M}_{0} \times \dot{\boldsymbol{B}}_{\mathrm{Str}},
$$

which is similar to Eq. (23) but with $\boldsymbol{E}$ replaced by $\dot{\boldsymbol{B}}_{\text {Str }}$. This systematic effect can be monitored by sensitive magnetometry and discriminated, for instance, by its relative phase.

\section{CONCLUSIONS}

In conclusion, the nuclear-spin-dependent paritynonconserving contribution to the $J$-coupling Hamiltonian in diatomic molecules puts observation of molecular PNC within the reach of current atomic vapor-cell magnetometers, even for molecules containing relatively light atoms such as HF. A detailed analysis of systematic effects will need to be carried out in conjunction with a specific experimental design. Many potentially dangerous systematic effects can be discriminated based on several available reversals: that of the initial magnetization, detection direction, and the phase of the applied electric-field modulation. Additional tell-tale features of the useful signal include the fact that the signal appears at a frequency different from the frequency with which the electric field is modulated; moreover, the PNC-signal frequency scales as the square of the amplitude of the applied field [see Eq. (20)].

For simplicity we considered diatomic molecules as an example. However, this method should work also for more complicated a chiral polar molecules. The size of the respective PNC effect can be estimated using Eq. (15). For molecules with spin- $1 / 2$ nuclei in the range $7 \leqslant Z \leqslant 81$, the PNC coupling is likely to be between a few microhertz and a few millihertz, as suggested by the estimates (17) and (16).

\section{ACKNOWLEDGMENTS}

This work was supported by the National Science Foundation under Grant No. CHE-1709944 and by the Cluster of Excellence "Precision Physics, Fundamental Interactions, and Structure of Matter" (PRISMA+ EXC 2118/1), funded by the German Research Foundation (DFG) within the German Excellence Strategy (Project ID 39083149). M.G.K. is grateful to the Mainz Institute for Theoretical Physics (MITP) for its hospitality and acknowledges support from Russian Science Foundation under Grant No. 19-12-00157. The authors wish to acknowledge Prof. Robert Harris for useful discussions on symmetry and parity nonconservation, Prof. David DeMille for his comments on the theory and possible experimental implementations, and Dr. Leonid Skripnikov for finding a numerical error in our estimates.
[1] T. D. Lee and C. N. Yang, Question of parity conservation in weak interactions, Phys. Rev. 104, 254 (1956).

[2] C. S. Wu, E. Ambler, R. W. Hayward, D. D. Hoppes, and R. P. Hudson, Experimental test of parity conservation in beta decay, Phys. Rev. 105, 1413 (1957).

[3] M-.A. Bouchiat, Atomic parity violation: Early days, present results, prospects, Il Nuovo Cimento C 35, 78 (2012).

[4] M. S. Safronova, D. Budker, D. DeMille, D. F. Jackson Kimball, A. Derevianko, and C. W. Clark, Search for new physics with atoms and molecules, Rev. Mod. Phys. 90, 025008 (2018).

[5] J. S. M. Ginges and Victor V. Flambaum, Violations of fundamental symmetries in atoms and tests of unification theories of elementary particles, Phys. Rep. 397, 63 (2004).

[6] R. Berger and J. Stohner, Parity violation, WIREs Comput. Mol. Sci. 9, e1396 (2019).

[7] E. Gajzago and G. Marx, Weak energy difference between mirror molecules, Atomki Kozl. Suppl. 16, 177 (1974).

[8] E. Gajzago and G. Marx, On the weak energy difference between mirror molecules, in Fourth International Conference on Physics and Astrophysics NEUTRINOS - 1974, edited by H. C. Wolfe and C. Baltay, AIP Conf. Proc. No. 22 (AIP, New York, 1974), p. 93.

[9] V. S. Letokhov, On difference of energy levels of left and right molecules due to weak interactions, Phys. Lett. A 53, 275 (1975).
[10] O. N. Kompanets, A. R. Kukudzhanov, V. S. Letokhov, and L. L. Gervits, Narrow resonances of saturated absorption of the asymmetrical molecule $\mathrm{CHFClBr}$ and the possibility of weak current detection in molecular physics, Opt. Commun. 19, 414 (1976).

[11] Robert A. Harris and Leo Stodolsky, The effect of the parity violating electron-nucleus interaction on the spin-spin coupling Hamiltonian of chiral molecules, J. Chem. Phys. 73, 3862 (1980).

[12] V. G. Gorshkov, M. G. Kozlov, and L. N. Labzovskii, P-odd effects in polyatomic molecules, Sov. Phys. JETP 55, 1042 (1982); P-odd effects in polyatomic molecules [Zh. Eksp. Teor. Fiz. 82, 1807 (1982)].

[13] A. Bauder, A. Beil, D. Luckhaus, F. Müller, and M. Quack, Combined high resolution infrared and microwave study of bromochlorofluoromethane, J. Chem. Phys 106, 7558 (1997).

[14] A. S. Lahamer, S. M. Mahurin, R. N. Compton, D. House, J. K. Laerdahl, M. Lein, and P. Schwerdtfeger, Search for a ParityViolating Energy Difference between Enantiomers of a Chiral Iron Complex, Phys. Rev. Lett 85, 4470 (2000).

[15] B. Darquie, C. Stoeffler, S. Zrig, J. Crassous, P. Soulard, P. Asselin, T. R. Huet, L. Guy, R. Bast, T. Saue, P. Schwerdtfeger, A. Shelkovnikov, and C. Chardonnet, Progress toward the first observation of parity violation in chiral molecules by highresolution laser spectroscopy, Chirality 22, 870 (2010). 
[16] J. Crassous, F. Monier, J. P. Dutasta, M. Ziskind, C. Daussy, C. Grain, and C. Chardonnet, Search for resolution of chiral fluorohalogenomethanes and parity-violation effects at the molecular level, ChemPhysChem 4, 541 (2003).

[17] A. Cournol, M. Manceau, M. Pierens, L. Lecordier, D. B. A. Tran, R. Santagata, B. Argence, A. Goncharov, O. Lopez, M. Abgrall, Y. Le Coq, R. Le Targat, H. Alvarez Martinez, W. K. Lee, D. Xu, P. E. Pottie, R. J. Hendricks, T. E. Wall, J. M. Bieniewska, B. E. Sauer et al., A new experiment to test parity symmetry in cold chiral molecules using vibrational spectroscopy, Quantum Electron. 49, 288 (2019).

[18] R. A. Harris and L. Stodolsky, Quantum beats in optical activity and weak interactions, Phys. Lett. B 78, 313 (1978).

[19] A. Szabó-Nagy and L. Keszthelyi, Demonstration of the parityviolating energy difference between enantiomers, Proc. Natl. Acad. Sci. U.S.A 96, 4252 (1999).

[20] A. J. MacDermott and R. A. Hegstrom, A proposed experiment to measure the parity-violating energy difference between enantiomers from the optical rotation of chiral ammonia-like "cat" molecules, Chem. Phys. 305, 55 (2004).

[21] D. DeMille, S. B. Cahn, D. Murphree, D. A. Rahmlow, and M. G. Kozlov, Using Molecules to Measure Nuclear SpinDependent Parity Violation, Phys. Rev. Lett 100, 023003 (2008).

[22] S. B. Cahn, J. Ammon, E. Kirilov, Y. V. Gurevich, D. Murphree, R. Paolino, D. A. Rahmlow, M. G. Kozlov, and D. DeMille, Zeeman-Tuned Rotational Level-Crossing Spectroscopy in a Diatomic Free Radical, Phys. Rev. Lett 112, 163002 (2014).

[23] E. Altuntas, J. Ammon, S. B. Cahn, and D. DeMille, Demonstration of a Sensitive Method to Measure Nuclear SpinDependent Parity Violation, Phys. Rev. Lett. 120, 142501 (2018).

[24] V. V. Flambaum and I. B. Khriplovich, On the enhancement of parity nonconserving effects in diatomic molecules, Phys. Lett. A 110, 121 (1985).

[25] M.G. Kozlov and L. N. Labzowsky, Parity violation effects in diatomics, J. Phys. B 28, 1933 (1995).

[26] I. B. Khriplovich, Parity Non-Conservation in Atomic Phenomena (Gordon and Breach, New York, 1991).

[27] A. L. Barra and J. B. Robert, Parity non-conservation and NMR parameters, Mol. Phys. 88, 875 (1996).

[28] J.-B. Robert and A. L. Barra, NMR and parity nonconservation, Experimental requirements to observe a difference between enantiomer signals, Chirality 13, 699 (2001).

[29] G. Laubender and R. Berger, Ab initio calculation of parityviolating chemical shifts in NMR spectra of chiral molecules, ChemPhysChem 4, 395 (2003).

[30] V. Weijo, P. Manninen, and J. Vaara, Perturbational calculations of parity-violating effects in nuclear-magnetic-resonance parameters, J. Chem. Phys. 123, 054501 (2005).

[31] G. Laubender and R. Berger, Electroweak quantum chemistry for nuclear-magnetic-resonance-shielding constants: Impact of electron correlation, Phys. Rev. A 74, 032105 (2006).

[32] Radovan Bast, Peter Schwerdtfeger, and Trond Saue, Parity nonconservation contribution to the nuclear magnetic resonance shielding constants of chiral molecules: A four-component relativistic study, J. Chem. Phys. 125, 064504 (2006).

[33] V. Weijo, R. Bast, P. Manninen, T. Saue, and J. Vaara, Methodological aspects in the calculation of parity-violating effects in nuclear magnetic resonance parameters, J. Chem. Phys. 126, 074107 (2007).

[34] S. Nahrwold and R. Berger, Zeroth order regular approximation approach to parity violating nuclear magnetic resonance shielding tensors, J. Chem. Phys. 130, 214101 (2009).

[35] Sophie Nahrwold, Robert Berger, and Peter Schwerdtfeger, Parity violation in nuclear magnetic resonance frequencies of chiral tetrahedral tungsten complexes NWXYZ (X, Y, Z = H, $\mathrm{F}, \mathrm{Cl}, \mathrm{Br}$ or I), J. Chem. Phys. 140, 024305 (2014).

[36] J. Eills, J. W. Blanchard, L. Bougas, M. G. Kozlov, A. Pines, and D. Budker, Measuring molecular parity nonconservation using nuclear-magnetic-resonance spectroscopy, Phys. Rev. A 96, 042119 (2017)

[37] H. S. Gutowsky, D. W. McCall, and C. P. Slichter, Coupling among nuclear magnetic dipoles in molecules, Phys. Rev. 84, 589 (1951).

[38] E. L. Hahn and D. E. Maxwell, Spin echo measurements of nuclear spin coupling in molecules, Phys. Rev. 88, 1070 (1952).

[39] N. F. Ramsey, Electron coupled interactions between nuclear spins in molecules, Phys. Rev. 91, 303 (1953).

[40] P. Garbacz and A. D. Buckingham, Chirality-sensitive nuclear magnetic resonance effects induced by indirect spin-spin coupling, J. Chem. Phys. 145, 204201 (2016).

[41] J. P. King, T. F. Sjolander, and J. W. Blanchard, Antisymmetric couplings enable direct observation of chirality in nuclear magnetic resonance spectroscopy, J. Phys. Chem. Lett. 8, 710 (2017).

[42] See Supplemental Material at http://link.aps.org/supplemental/ 10.1103/PhysRevResearch.2.023258 for a short discussion of symmetry rules for rank- $1 J$ couplings and further details for the relativistic enhancement factor of Eq. (14).

[43] V. V. Flambaum and I. B. Khriplovich, New restrictions on the electric dipole moment of the electron and t-odd electronnucleon interaction, Sov. Phys. JETP 62, 872 (1985).

[44] C. Bouchiat and C. A. Piketty, Nuclear spin dependent parity violating electron-nucleus interaction in heavy atoms, The anapole moment and the perturbation of the Hadronic vector neutral current by the hyperfine interaction, Phys. Lett. B 269, 195 (1991); 274, 526(E) (1992).

[45] V. V. Flambaum and I. B. Khriplovich, P-odd nuclear forces as a source of parity nonconservation in atoms, Sov. Phys.-JETP 52, 835 (1980).

[46] V. V. Flambaum, I. B. Khriplovich, and O. P. Sushkov, Nuclear anapole moments, Phys. Lett. B 146, 367 (1984).

[47] V. V .Flambaum and D. W. Murray, Anapole moment and nucleon weak interaction, Phys. Rev. C 56, 1641 (1997).

[48] G. A. Aucar, T. Saue, L. Visscher, and H. J. Aa. Jensen, On the origin and contribution of the diamagnetic term in fourcomponent relativistic calculations of magnetic properties, J. Chem. Phys. 110, 6208 (1999).

[49] I. I. Sobelman, Atomic Spectra and Radiative Transitions (Springer-Verlag, Berlin, 1979).

[50] M. A. Bouchiat and C. Bouchiat, Parity violation induced by weak neutral currents in atomic physics, Phys. Lett. B 48, 111 (1974).

[51] E. A. Hinds and P. G. H. Sandars, Electric dipole hyperfine structure of TIF, Phys. Rev. A 21, 471 (1980).

[52] D. Cho, K. Sangster, and E. A. Hinds, Search for time-reversalsymmetry violation in thallium fluoride using a jet source, Phys. Rev. A 44, 2783 (1991). 
[53] V. L. Varentsov, V. G. Gorshkov, V. F. Ezhov, M. G. Kozlov, L. N. Labzovskii, and V. N. Fomichev, Possible P,T-odd effects in NMR spectroscopy of molecules, JETP Lett. 36, 175 (1982) [Pis'ma Zh. Eksp. Teor. Fiz. 36, 141 (1982)].

[54] E. B. Norrgard, E. R. Edwards, D. J. McCarron, M. H. Steinecker, D. DeMille, S. S. Alam, S. K. Peck, N. S. Wadia, and L. R. Hunter, Hyperfine structure of the $\mathrm{B}^{3} \Pi_{1}$ state and predictions of optical cycling behavior in the $\mathrm{X} \rightarrow \mathrm{B}$ transition of TIF, Phys. Rev. A 95, 062506 (2017).

[55] O. Grasdijk, D. DeMille, J. Kastelic, D. Kawall, S. Lamoreaux, O. Timgren, K. Wenz, T. Winick, T. Wright, and T. Zelevinsky, Search for parity-and time reversal-violating nuclear spindependent interactions in ${ }^{205} \mathrm{TlF}$, Bull. Am. Phys. Soc. (2019).

[56] R. v. Boeckh, G. Gräff, and R. Ley, Die Abhängigkeit innerer und äußerer Wechselwirkungen des TIF-Moleküls von der Schwingung, Rotation und Isotopie, Z. Physik 179, 285 (1964).

[57] D. L. Bryce and R. E. Wasylishen, Indirect nuclear spin-spin coupling tensors in diatomic molecules: A comparison of results obtained by experiment and first principles calculations, J. Am. Chem. Soc. 122, 3197 (2000).

[58] J. Shamir and A. Netzer, NMR studies of anhydrous hydrogen fluoride solutions, Can. J. Chem. 51, 2676 (1973).

[59] J. S. Martin and F. Y. Fujiwara, High resolution nuclear magnetic resonance spectra of hydrogen fluoride in solution and in bihalide ions, Nuclear spin coupling in strong hydrogen bonds, J. Am. Chem. Soc. 96, 7632 (1974).

[60] E. L. Mackor, C. MacLean, and C. W. Hilbers, NMR of hydrogen fluoride in the gas phase, Recueil des Travaux Chimiques des Pays-Bas 87, 655 (1968).

[61] A. Krachmalnicoff, R. Bounds, S. Mamone, S. Alom, M. Concistrè, B. Meier, K. Kouřil, M. E. Light, M. R Johnson, S. Rols, A. J. Horsewill, A. Shugai, U. Nagel, T. Rõõm, M. Carravetta, M. H. Levitt, and R. J. Whitby, The dipolar endofullerene HF@C60, Nat. Chem. 8, 953 (2016).

[62] A. D. Buckingham and J. A. Pople, High-resolution N.M.R. spectra, J. Chem. Soc. Faraday Trans. 59, 2421 (1963).
[63] S. A. Riley and M. P. Augustine, Extracting residual NMR coupling constants from electrically aligned liquids, J. Phys. Chem. A 104, 3326 (2000).

[64] J. W. Blanchard and D. Budker, Zero- to ultralow-field NMR, eMagRes 5, 1395 (2017).

[65] J. S. Muenter and William Klemperer, Hyperfine structure constants of HF and DF, J. Chem. Phys. 52, 6033 (1970).

[66] J. W. Blanchard, T. F. Sjolander, J. P. King, M. P. Ledbetter, E. H. Levine, V. S. Bajaj, D. Budker, and A. Pines, Measurement of untruncated nuclear spin interactions via zero- to ultralow-field nuclear magnetic resonance, Phys. Rev. B 92, 220202(R) (2015).

[67] M. Auzinsh, D. Budker, and S. Rochester, Optically Polarized Atoms: Understanding Light-Atom Interactions (Oxford University Press, Oxford, England, 2010).

[68] James Keeler, Understanding NMR Spectroscopy (John Wiley \& Sons, New York, 2011).

[69] M. C. D. Tayler, T. F. Sjolander, A. Pines, and D. Budker, Nuclear magnetic resonance at millitesla fields using a zerofield spectrometer, J. Magn. Reson. 270, 35 (2016).

[70] M. Jiang, T. Wu, J. W. Blanchard, G. Feng, X. Peng, and D. Budker, Experimental benchmarking of quantum control in zero-field nuclear magnetic resonance, Sci. Adv. 4, eaar6327 (2018).

[71] M. C. D. Tayler, T. Theis, T. F. Sjolander, J. W. Blanchard, A. Kentner, S. Pustelny, A. Pines, and D. Budker, Invited review article: Instrumentation for nuclear magnetic resonance in zero and ultralow magnetic field, Rev. Sci. Instrum. 88, 091101 (2017).

[72] T. Wu, J. W. Blanchard, G. P. Centers, N. L. Figueroa, A. Garcon, P. W. Graham, D. F. Jackson Kimball, S. Rajendran, Y. V. Stadnik, A. O. Sushkov, A. Wickenbrock, and D. Budker, Search for Axionlike Dark Matter with a Liquid-State $\mathrm{Nu}$ clear Spin Comagnetometer, Phys. Rev. Lett. 122, 191302 (2019). 